\title{
Enhancing students' experimental knowledge with active learning in a pharmaceutical science laboratory
}

\author{
Megan Anakin ${ }^{1}$ (D) , Arlene McDowell2 \\ ${ }^{1}$ Education Unit, Dunedin School of Medicine, University of Otago, Dunedin, New Zealand \\ ${ }^{2} S$ chool of Pharmacy, University of Otago, Dunedin, New Zealand
}

\author{
Keywords \\ Active learning \\ Pharmaceutical science \\ Experimental knowledge \\ Laboratory experiment \\ Pharmacy education \\ Correspondence \\ Dr Megan Anakin \\ Education Unit \\ Dean's Office \\ Dunedin School of Medicine \\ University of Otago \\ PO Box 56 \\ Dunedin \\ New Zealand 9054 \\ megan.anakin@otago.ac.nz
}

\begin{abstract}
Objective: The study aimed to examine if an active learning approach used in a pharmaceutical science laboratory would enhance pharmacy students' learning of foundation pharmaceutical science knowledge when conducting an experiment. Method: A pre-post-test study design was used to collect data from third-year undergraduate pharmacy students with two approaches to performing an experiment (active learning, and traditional). Results: Assessment data from 95 students (73\% response rate) were analysed quantitatively and qualitatively. The active learning approach to performing an experiment resulted in significantly higher $(p<0.001)$ scores compared to the traditional approach for knowledge about the variables to be measured (3.82 versus 2.72 for active and traditional, respectively) and measurement method (3.31 versus 2.85 for active and traditional, respectively). A thematic analysis identified 'planning' as unique to the post-test responses for the active learning session. Conclusion: The authors concluded that the laboratory session featuring active learning had a greater impact on student learning than the traditional experiment method.
\end{abstract}

\section{Introduction}

Active learning is an educational concept about instructional practice where students engage in the learning process by performing sense-making activities, such as discussions and problem solving, rather than passively receiving knowledge transmitted to them from their teachers or by working with course materials alone (Sfard, 1998). In this article, we distinguish between the term active learning as an educational concept and the active learning that may occur during a science laboratory class. A laboratory class may be viewed as an active learning environment because it typically involves performing an aim-oriented set of procedures with specialised equipment and writing a report about findings (Gleason et al.,
2011). These two versions of active learning may be mutually inclusive when students are invited to design and discuss their own experimental protocol to perform an experiment to solve a given problem rather than follow step-by-step instructions and procedures listed in a manual (Thompson, Caroll, \& Laibe, 2013).

Educators in higher education science contexts have been exploring how the educational concept of active learning can be applied to laboratory sessions. Recent efforts to study the benefits active learning include a meta-analysis which summarises research evidence that supports the use of active learning approaches as the preferred teaching practice in undergraduate science, technology, engineering, and mathematics courses (Freeman, 2014). More 
specifically in the field of biology, a combination of interactive lectures and digital resources were the active learning strategies that significantly improved students' grades in a three-week molecular biology course (Scott et al., 2018). Likewise, when biochemistry and molecular biology laboratory sessions were redesigned to depart from a recipe approach, the new active learning format was acceptable to students and resulted in measurable differences in student achievement related to learning outcomes for each session (Arthur et al., 2016). In chemistry laboratory sessions, active learning approaches that include guided inquiry and independent group projects were found to increase assessment results for specific learning outcomes related to fundamental concepts such as sampling and instrument calibration (Cavinato, 2017). These examples demonstrate the effectiveness of an active learning approach to address student-level factors such as acceptability, motivation, and interest as well as learning-level outcomes such as course grades and assessments of fundamental scientific concepts.

Recent studies published in pharmacy education report the effectiveness of active learning strategies on student learning of pharmacy practice skills in laboratory settings or pharmaceutical science knowledge in lectures, but not in laboratory sessions (Kangwantas et al., 2017; Meng et al., 2019). For example, Kiersma and colleagues found that an active learning laboratory session that used simulations helped first-year pharmacy students improve their knowledge and skills to detect and resolve medication errors (Kiersma et al., 2009). Likewise, a design project using an active learning strategy was used in laboratory and experiential settings to enhance students' early professional practice experiences and decision-making activities (Ross et al., 1999). Missing from this literature are studies that explore students' foundation knowledge in pharmaceutical science laboratory settings that involve conducting an experiment.

Evidence about the effectiveness of using an active learning approach to develop student knowledge of how to conduct an experiment in a pharmaceutical science laboratory setting is also lacking from the literature. Studies are needed to help pharmacy educators to better understand how they can enhance their pharmacy programmes to meet accreditation standards that relate to providing a quality learning environment, and producing suitably knowledgeable and skilled practitioners with foundational concepts in pharmaceutical sciences. For example, in the United States of America, the accreditation standards specify:

'Key Element 1.1. Foundational knowledge - The graduate is able to develop, integrate, and apply knowledge from the foundational sciences (i.e., biomedical, pharmaceutical, social/behavioral/administrative, and clinical sciences) to evaluate the scientific literature, explain drug action, solve therapeutic problems, and advance population health and patient-centred care'

[Accreditation Council for Pharmacy Education, 2016: p.1]

In Australia and New Zealand, one performance outcome in the accreditation framework related to foundation science knowledge is:

'5.4 demonstrating knowledge and skills in research and inquiry, including a. formulating questions $b$. identifying and critically appraising relevant source materials c. undertaking relevant investigations, where appropriate $d$. drawing conclusions by synthesising the results of research and inquiry activities e. reporting and disseminating the outcomes appropriately $f$. identifying ways in which the outcomes can be applied to practice'

[Australian Pharmacy Council, 2020: p.22]

These outcome expectations require students to develop a thorough understanding of foundational pharmaceutical knowledge because pharmacy is a science-based profession (International Pharmaceutical Federation, 2016; International Pharmaceutical Federation, 2017). To provide a breath of understanding as future practicing pharmacists, we contend that this foundation will be enhanced with knowledge of how to conduct an experiment in a pharmaceutical science laboratory setting.

One promising approach that may be successfully adapted to enhance the foundation sciences knowledge of pharmacy students about conducting science experiments is reported by Thompson and colleagues (Thompson, Caroll, \& Laibe, 2013; Thompson et al., 2014). They have implemented an inquiry-oriented active learning approach that had a positive impact of first-year chemistry students' perceptions of their critical thinking and investigative skills when conducting an experiment in a laboratory setting. To date, this active learning approach has not been investigated further to measure its impact on student learning of foundational pharmaceutical science knowledge in pharmacy education. Therefore, this study aimed to examine if an active learning approach used in a pharmaceutical science laboratory setting would enhance pharmacy students' learning about foundation pharmaceutical science knowledge about conducting an experiment. 


\section{Methods}

This study used a mixed methods approach (Creswell \& Creswell, 2018) to address the following research question - when students conduct a scientific experiment during a pharmaceutical laboratory class, does an active learning approach result in greater learning than a traditional laboratory class? The study design was approved by the University of Otago Human Ethics Committee (D17/100). The significance of this study for Māori student learning and Māori health workforce development was discussed with the Ngai Tāhu Research Consultation Committee. Students that self-identify as Māori are under-represented in pharmacy education and practice. Consequently, consultation with Māori about the features of the study is a cultural imperative in New Zealand. This study was conducted at the School of Pharmacy at the University of Otago, Dunedin, New Zealand where students complete a fouryear programme to be awarded a Bachelor of Pharmacy. Experimental knowledge is primarily learned in the first three years of their degree; whereas, in their fourth-year, students are expected to apply their experimental knowledge to clinical practice.

The participants in this study were third-year undergraduate students who were enrolled in the course titled ' $\mathrm{PH}$ CY342: Drug Delivery Systems'. This 13-week course took place from March to June 2017 and it consisted of 32 lectures, five three-hour laboratory sessions, and one tutorial class. Lectures were presented to all students $(n=130)$ as one group, whereas, laboratory sessions were conducted with students divided into four equal groups (approximately $n=33$ ). Each group was scheduled to perform their laboratory experiment during a different three-hour session in the same laboratory within a two week period. Consequently, the interval between laboratory sessions could be up to two weeks for any group of students. The first two laboratory classes for the course provided students with the opportunity to become familiar with the equipment and techniques. Students could then build on their experiences to plan their own experiment for the active learning experiment in the third laboratory class. The topic of the third laboratory class also provided scope for four variables to be explored by students. The fourth class was a traditional lab format where students followed a list of instructions. Data for this study were collected during the third and fourth laboratory classes.

In this study, the term 'experimental knowledge' refers to the abstract ideas a scientist must understand and apply correctly in order to perform and interpret the results of a scientific experiment according to accepted norms and conventions (Schiemann, 2006). The two independent variables in this study were teaching method (active learn- ing, traditional) and timing of the questionnaire (pre-lab, post-lab). The active learning experiment method was developed from of the 'IDEA experiment' developed by Thompson and colleagues and it required students to design, plan, and perform their own experiment (Thompson, Caroll, \& Laibe, 2013; Thompson et al., 2014). The traditional experiment method was used for comparison and it involved a verbal introduction to the topic of the laboratory class and students following a detailed list of instructions in the course laboratory manual with instructions on what activity to perform at each step. For the active learning experiment, in a lecture prior to the lab class, students were told that they would be designing their own experiment to explore a research question. As for the traditional experiment, a brief introduction was given about the lab and students then worked in groups to design their experiment. A lab class of approximately 33 students was supervised by three demonstrators (postgraduate students) and one academic staff member who circulated during the class to provide some guidance to students while they were designing their experiment and performing the practical activities.

All students enrolled in PHCY342 ( $n=130)$ were invited to participate in the study. To avoid students feeling coerced by their lecturer inviting them to participate in the study, the second author focused on teaching and the first author performed participant recruitment and data collection. Participants were informed that they were welcome to withdraw from the study up to the time that the data were analysed, and they would receive no incentive for participating; Participants were reminded that their participation was voluntary. They were also reminded that all identifying information would be removed from their questionnaires and they would be assigned a random number designation (i.e. P1, P2, P3) by the first author to protect their anonymity. Data were collected from students during two consecutive laboratory classes. The active learning experiment method was used during the third laboratory session in the course and involved measuring dissolution to investigate the effect of excipients (hydroxypropyl cellulose and stearic acid at 5\%, 10\%, 20\% or $40 \%$ ) on drug release. See Appendix A to view the experiment instructions given to students in the laboratory manual for the third experiment before and after, they were revised to feature the active learning experiment method. The traditional experiment method was used during the subsequent laboratory session and involved observing the disintegration of enteric coated tablets in buffers with different $\mathrm{pH}$.

To investigate the impact of each teaching method as an independent variable (active learning, traditional), six 
questions were written by the authors, one of whom has expertise in pharmaceutical sciences and the other with expertise in educational research, to operationalise the foundational pharmaceutical science knowledge specified in the programmatic outcomes of accreditation (Accreditation Council for Pharmacy Education, 2016; Australian Pharmacy Council, 2020). The first four questions collected assessment data about the experiment's purpose (Question 1), variables to be measured (Question 2), measurement method (Question 3), and measurement reasonableness (Question 4). These finer grained assessment data were collected because the laboratory reports were marked as pass or fail and all students passed both reports, therefore, other assessment data were not suitable for use in this study. The last two questions collected data about students' perceptions of learning. They were about learning expectations (Questions 5) and future actions (Question 6).

Two parallel versions of the questions were written for data collection before and after each laboratory session to account for timing (pre-lab, post-lab) as an additional independent variable. The questions used are shown in Figure I.
To analyse the data from the questionnaires, a research assistant transcribed the anonymised written responses into an Excel spreadsheet for independent coding by the authors. Scoring criteria for Questions 1-4 were developed from students' written responses by applying the Structure of Observed Learning Outcomes (SOLO) framework (Biggs \& Collis, 1982) to them. The SOLO framework enabled the authors to describe levels of increasing complexity in students' understanding of the foundation pharmaceutical science knowledge. Criteria were developed by each author working independently to identify examples at each point of a six-point scale as shown in the left column in Table I. The authors met to come to consensus on representative examples for each point in the scale to act as exemplars shown in the centre and right columns of Table I. These exemplars were used as anchor points when assigning a score to each response. The authors then worked independently to apply the scoring criteria to each response for Questions 1 to 4 . Inter-rater reliability was assessed using a two-way mixed effects model, absolute agreement, average measures intra-class correlation (Hallgren, 2012). The intra-class correlation coefficients were calculated using IBM, SPSS (Version 25 2018,

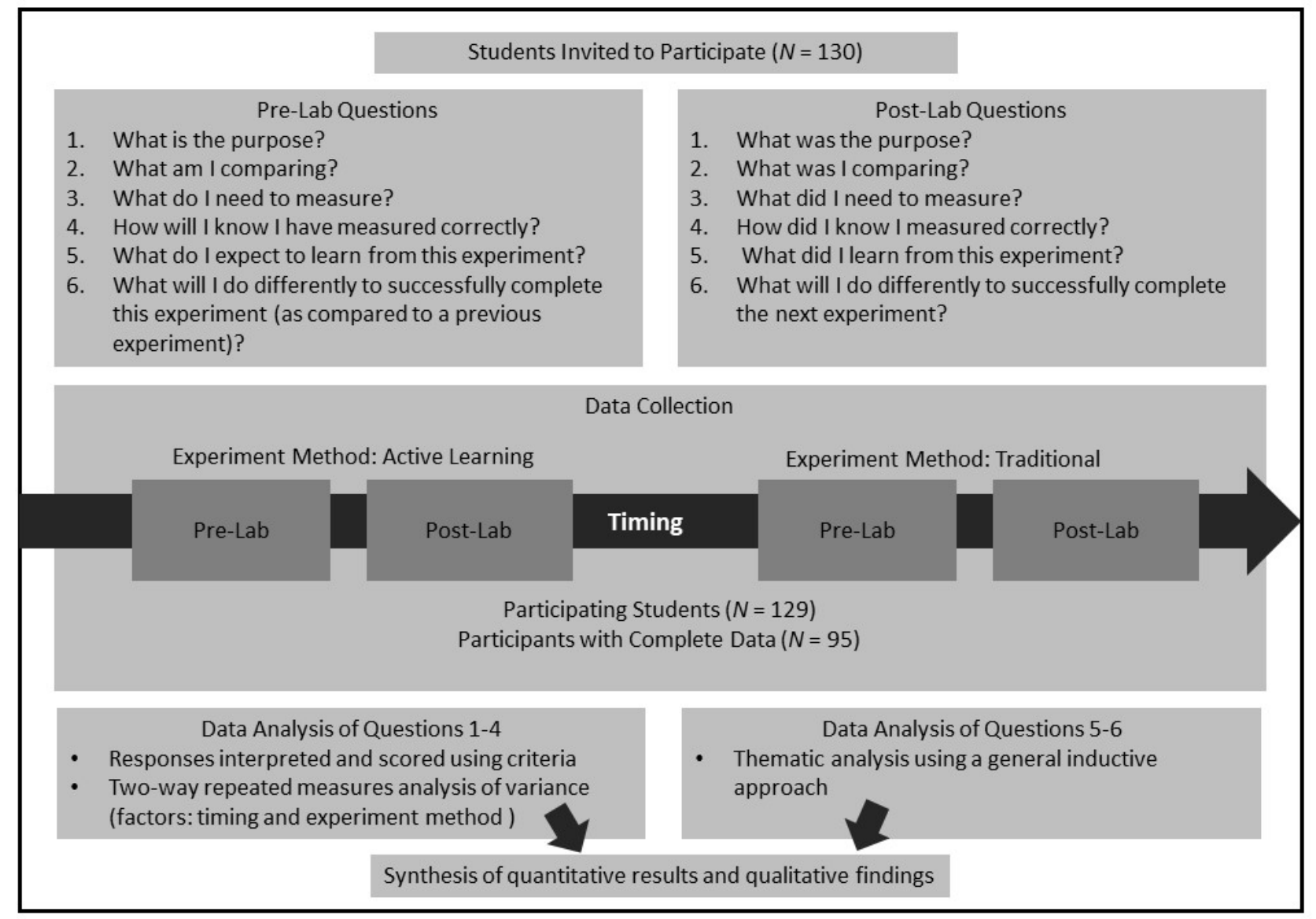

Figure I. An overview of the data collection and analysis procedures used in this study 
Table I: Scoring criteria with representative responses for questions 1 to 4 for the active learning laboratory session by timing of the questionnaire (Pre-Lab, Post-Lab)

\begin{tabular}{|c|c|c|c|}
\hline Score & Criteria & $\begin{array}{c}\text { Representative Response } \\
\text { (Pre-Lab) }\end{array}$ & $\begin{array}{l}\text { Representative Response } \\
\text { (Post-Lab) }\end{array}$ \\
\hline \multicolumn{4}{|c|}{ Question 1: What Is / Was the Purpose? } \\
\hline 5 & $\begin{array}{l}\text { A complete and succinct statement of the purpose of this experiment. Purpose } \\
\text { statement contains specific details about key components (e.g. excipients) AND } \\
\text { processes (dissolution rate) AND relationships to be investigated (different con- } \\
\text { centrations of excipients) }\end{array}$ & $\begin{array}{l}\text { 'To design an experiment on measuring the } \\
\text { dissolution rate of theophylline with differ- } \\
\text { ent concentrations of excipients being } \\
\text { hydroxy-propylcellulose and stearic }\end{array}$ & $\begin{array}{l}\text { 'To determine the dis-solution rate } \\
\text { of theo-phylline with different } \\
\text { concentration of excipient HPC \& } \\
\text { SA' [P25] }\end{array}$ \\
\hline 4 & $\begin{array}{l}\text { Purpose statement contains specific details about key components (e.g. excipi- } \\
\text { ents) AND processes (dissolution rate) OR relationships to be investigated (differ- } \\
\text { ent concentrations of excipients) }\end{array}$ & $\begin{array}{l}\text { 'To study the effects of hydroxypropyl cellu- } \\
\text { lose and stearic acid on the dissolution rate } \\
\text { of theophylline' [PP25] }\end{array}$ & $\begin{array}{l}\text { 'To test dissolution with changing } \\
\text { concentrations of HPC and } \\
\text { SA' [P86] }\end{array}$ \\
\hline
\end{tabular}

General purpose of this experiment is described. May contain specific details

3 about key components (e.g. excipients) OR processes (dissolution rate) OR relationships to be investigated (different concentrations of excipients)

Purpose statement about experimen-tation but not specific enough to this experiment

1 Engaged with the question

0 No response

'Determining the effect excipients have on dissolution rates' $[\mathrm{P} 72]$

'To investigate the effect of excipi-

'To compare two controlled release ents on drug release' [P9]

tablets' [P34]

II don't know' [P9]

[P40]

'To compare exci-pients' [P64]

Nil

[P41]

Question 2: What Am / Was I Comparing?

A complete and succinct statement of the comparisons in this experiment.

Statement contains specific details about the TWO variables (e.g. excipients)

AND (different concentrations of excipients) AND specific processes of dissolution (dissolution rate of theophylline)

Comparison statement contains specific details about TWO variables identified

4 (e.g. excipients) AND relationships to be investigated (different concentrations of excipients)

'Comparing the effect of adding different concentrations of hydroxypropyl cellulose (HPC) and stearic acid on the dissolution rate of theophylline' [P21]

'To investigate the effects of hydroxypropyl cellulose and stearic acids on the dissolution rate of theophylline' [P92]

Comparison statement of this experiment is described. Comparison may be implied because 2 excipients are named (e.g. vs). One variable has been identi-

3 fied. (e.g. excipients) OR relationships to be investigated (different concentrations of excipients)

2 Comparison statement about dissolution but not specific enough to the excipi-

ents in this experiment. Variables not explicitly identified

Engaged with the question

0 No response

Question 3: What Do / Did I N

5 Identify THREE parameters to be measured and their relationship absorbance/ concentration, dissolution rate, and kinetics/over time / rate

4 Identify TWO parameters to be measured and their relationship from the following absorbance/concentration, dissolution rate, and kinetics/over time/rate

Identified one parameter to be measured, but may be in isolation. May contain specific details about key components (e.g. absorbance / concentration) OR processes (dissolution rate) OR relationships to be investigated (kinetics / over time/rate)

2

Identified some parameter to be measured, but not specific enough to the purpose of the experiment

1 Engaged with the question

0 No response

\section{'Dis}

'Dissolution rate' [P41]
'Not sure' [P2]
[P84]

[P84]

'Dissolution of theophylline in presence $\Delta$ absence of hydroxypropyl cellulose \& stearic acid' [P84]

'Concentration of theophylline in medium at different time points' [P22]

'Concentration of drug dissolved in solution' [P57]

'Medium, temp, amount of excipients' [P18]

'I don't know' [P85]

[P87]

Question 4: How Will / Did I Know I Measured it Correctly?

Justification is based on the knowledge specific to this experiment and justifica-

5 tion contains specific details about two or more key parameters (absorbance) concentration, dissolution rate, and kinetics/over time/ rate)

Nil

Justification is based on the knowledge specific to this experiment and justifica-

4 tion contains specific details about ONE key parameter (absorbance/concentration, dissolution rate, and kinetics/over time/rate)

General measurements of this experiment is described. May contain specific

details about key components (e.g. absorbance/concentration/different excipi-

3 ents) OR processes (dissolution rate) OR relationships to be investigated (kinetics/over time/rate)

2 Measuring correctly is stated, but not justified. And may not be specific enough to this experiment

1 Engaged with the question

0 No response
'Dissolution rate look appropriate? Different rate for each variation' [P49]

'If the dissolution rates are consistent with the pharmacopeia monograph' [P38]

'Compare results to literature' [P56]

'Unsure' [P53]

[P72]
Different concentration of stearic acid \& how it affects dissolution of theophyl-line' [P15]

'Dissolution rate of the tablet with different \% of HPC' [P92]

'Stearic acid and hydroxyl cellulose' [P65]

'Dissolution rate' [P86]

Nil

[P41]

'The amount of drug release over time between tablets with different concentration of HPC' [P48] 'Dissolution rate \& absorbance of samples at diff times of dissolution' [P59]

'Dissolution rate' [P56]

'Amount of drug released into environment' [P68]

Nil

[P41]

'Highest percentage of excipients should show lowest dissolution rate/cumulative mass released for extended release' [P90]

'See a result where HPC \& SA cause in releasing of theophylline' [P19]

'By comparing the graphs of different concentrations of cu-mulative mass released' [P93]

'Check with demonstrator that we were doing to correctly' [P56]

'I don't' [P66]

[P41] 
Armonk, NY). The intra-class correlation coefficients ranged from 0.77 to 0.97 and were interpreted to have high reliability. Scores from these four questions were used as dependent variables in this study. Relationships among scores and the two independent variables (experiment method, timing) were explored using descriptive and inferential statistics. A two-way repeated measures analysis of variance was performed using SPSS for each of Questions 1 to 4 for the two laboratory sessions. The null hypothesis was that there is no significant difference between participants' scores for learning method and timing. An alpha level of 0.05 was used for all statistical tests. Since there are no other published accounts of statistical results for pharmacy students' experimental knowledge in a pharmaceutical science setting, the effect size benchmarks suggested by Cohen of 0.01 (small), 0.09 (medium), and 0.25 (large) were used for interpreting the partial eta squared results (Cohen, 1988).

Responses for Questions 5 and 6 were analysed by the authors using a General Inductive Approach (Thomas, 2006). This approach involved examining the transcribed comments and establishing links to features that students identified about their demonstrations of foundation pharmaceutical science knowledge when performing an experiment. This approach provided a straightforward method for analysing evaluation data. Coding was derived from the words or phrases used by students in their responses and only one code was applied to each response. Similar codes were grouped and a theme was identified when at least ten responses shared the same code.

\section{Results}

Of the 130 third-year students invited to participate, 129 students consented to participate in the study. The number of participants that completed all questions for both laboratory sessions was 95 (73\% response rate). In this sample, more participants were female (64 female, 31 male) and the majority identified as Asian or New Zealand European (39 Asian, 36 New Zealand European, 5 Māori, 25 other ethnicities - Note that frequencies in the data total more than 95 because participants can self-identify with more than one ethnicity). The gender and ethnicity of the sampled students was representative of the cohort of third-year students. There were no statistically significant relationships found among scores and demographic details.

A two-way repeated measures analysis of variance was conducted on the influence of two dependent variables (timing, learning method) on the average scores for the four experimental knowledge items. The means and standard deviations are shown in Table II.

Mean scores for all four experimental knowledge items showed significant differences with medium or large effect sizes for timing of the questionnaire (pre-lab, post-lab) (See Table III). The mean scores for questionnaires before the laboratory session were lower than the mean scores after the session for both experimental methods (active learning and traditional). This result was anticipated as learning was expected to occur during the laboratory session.

Table II: Descriptive statistics for experimental knowledge questionnaire scores $(n=95)$ by timing of the questionnaire (Pre-Lab, Post-Lab) and experiment method (Active Learning, Traditional)

\begin{tabular}{|c|c|c|c|c|c|c|c|c|}
\hline \multirow{3}{*}{$\begin{array}{l}\text { Questionnaire Item about } \\
\text { Experimental Knowledge }\end{array}$} & \multicolumn{4}{|c|}{ Pre-Lab } & \multicolumn{4}{|c|}{ Post-Lab } \\
\hline & \multicolumn{2}{|c|}{ Active Learning } & \multicolumn{2}{|c|}{ Traditional } & \multicolumn{2}{|c|}{ Active Learning } & \multicolumn{2}{|c|}{ Traditional } \\
\hline & $\mathbf{M}^{*}$ & SD** & $\mathbf{M}$ & SD & $\mathbf{M}$ & SD & $\mathbf{M}$ & SD \\
\hline 1. Purpose of the experiment & 3.16 & 0.891 & 3.25 & 0.978 & 3.81 & 0.878 & 3.39 & 0.734 \\
\hline 2. Variables to be Measured & 2.67 & 1.171 & 2.22 & 0.840 & 3.82 & 0.875 & 2.72 & 0.930 \\
\hline 3. Measurement Method & 2.94 & 0.965 & 2.27 & 0.791 & 3.31 & 0.670 & 2.85 & 0.771 \\
\hline 4. Measurement Reasonableness & 1.87 & 1.044 & 1.58 & 0.952 & 1.99 & 0.917 & 2.00 & 1.052 \\
\hline
\end{tabular}

* Mean score to three significant figures

** Standard deviation score to three significant figures

Note: Scores ranged from zero to five

Table III: Results of two-way Analysis of Variance for experimental knowledge questionnaire scores ( $n=95$ ) for main effects of timing of the questionnaire (Pre-Lab, Post-Lab), main effects of experiment method (Active Learning, Traditional), and interaction between timing and learning method

\begin{tabular}{|c|c|c|c|c|c|c|c|c|c|}
\hline \multirow{2}{*}{$\begin{array}{l}\text { Questionnaire Item about } \\
\text { Experimental Knowledge }\end{array}$} & \multicolumn{3}{|c|}{ Timing } & \multicolumn{3}{|c|}{ Experiment Method } & \multicolumn{3}{|c|}{ Timing and Experiment Method } \\
\hline & $F(1,94) *$ & $p$ & $\eta_{p^{2 * *}}$ & $F(1,94)$ & $p$ & $\eta_{p^{2}}$ & $F(1,94)$ & $p$ & $\eta_{p^{2}}$ \\
\hline 1. Purpose of the experiment & 19.340 & $<.001$ & 0.171 & 19.340 & 0.054 & $n s^{* * *}$ & 11.548 & $<.001$ & 0.109 \\
\hline 2. Variables to be Measured & 58.188 & $<.001$ & 0.382 & 82.273 & $<.001$ & 0.467 & 15.002 & $<.001$ & 0.138 \\
\hline 3. Measurement Method & 31.218 & $<.001$ & 0.249 & 47.978 & $<.001$ & 0.338 & 2.397 & 0.126 & ns \\
\hline 4. Measurement Reasonableness & 7.117 & 0.009 & 0.070 & 2.042 & 0.156 & ns & 3.013 & 0.086 & ns \\
\hline
\end{tabular}

*the $\mathrm{F}$ value from $\mathrm{F}$-degrees of freedom for two observations from 95 students.

** partial eta-squared (effect size measure)

$* * *$ not significant 
Mean scores for two experimental knowledge items, variables to be measured and measurement method, showed significant differences with large effects for experimental approach (active learning, traditional) (See Table III). The mean scores for questionnaires for the laboratory session with active learning were higher than the session with traditional experiment method. This result was interpreted cautiously to mean that students learned more about the variables to be measured, and measurement method during the laboratory session featuring the active learning approach than the traditional experiment method.

Mean scores for two experimental knowledge items, purpose of the experiment and variables to be measured, showed significant interactions with medium effect sizes (See Table III). Since there was no main effect, however, for purpose of the experiment, this result was not interpreted further. The mean scores for purpose of the experiment in the laboratory session with active learning were higher and had a greater magnitude of change after the session than for the traditional experiment. This result was cautiously interpreted to suggest that the laboratory session featuring active learning had a greater impact on student learning about variables to be measured than the traditional experiment method.

Four themes were identified in students' reflections: identifying variables, designing an experiment, preparation, and performing correct procedures (See Table IV). Two themes, preparation and identifying variables were identified in all four study conditions (pre-lab, post-lab, active learning, traditional). This finding suggests that to successfully manage and complete the experiments, whether using an active learning approach or traditional method, students were aware of the need to plan carefully and be aware of the features of the experiment that represented the variables they were studying. The theme 'performing correct procedures' was identified for all conditions except for in the questionnaire completed after the laboratory session using the traditional experiment. For the other three conditions, this theme was interpreted to suggest that students were concerned and interested in monitoring the procedures they were planning or performing. It is notable that the theme, designing an experiment, was identified in the pre-lab questionnaire data for the active learning laboratory session uniquely. This finding may indicate that students were aware that this approach to performing an experiment required them to think carefully about the components of the experiment they were about to perform and how they might monitor their progress.

\section{Discussion}

The results of this study were interpreted to suggest that active learning in a pharmaceutical science laboratory setting can be used to enhance students' experimental knowledge. As anticipated, students' written assessment responses to questions about experimental knowledge relevant to both laboratory sessions had higher average scores after performing each experiment than before. This observation supports the educational expectation that students will become more familiar with scientific concepts while performing an experiment (Accreditation Council for Pharmacy Education, 2016; Australian Pharmacy Council, 2020). In this study, regardless of whether an active learning approach or a traditional experimental method was used, students demonstrated enhanced knowledge about the purpose of the experiments they were conducting, the variables to be measured during the experiment, the measurement method they would use to measure the variables, and the reasonableness of their measurements. It is worth noting that measurement reasonableness had a lesser effect size compared to the other three experimental concepts. Measurement reasonableness might require more structured learning opportunities, as noted by Stallings and Gillmore (1971), because students often find it difficult to distinguish among measurement concepts such as accuracy, precision, reliability, and validity. Also of note, the scientific terms dissolution and disintegration were not always used correctly by students in their written comments about both experiments.

Also anticipated due to encouraging findings about active learning in pharmacy education (Kangwantas et al., 2017; Kiersma et al., 2009; Meng et al., 2019; Ross et al., 1999), students' assessment responses to two questions about experimental knowledge in the laboratory session featuring active learning had higher average scores than the session using the traditional approach. It appears that an active learning approach may be better than following a set list of procedures for learning about the variables to be measured during the experiment and understanding the measurement method used to measure the variables. This observation supports the benefits of using active learning as an instructional model that promotes students to participate actively in constructing their understanding of the experiment while performing it (Sfard, 1998). By using a version of the active learning approach proposed by Thompson and colleagues, this study adds to the limited literature about the impact of active learning on student learning in pharmaceutical science laboratory set- 
tings (Thompson, Caroll, \& Laibe, 2013; Thompson et al., 2014).

Additionally, an unanticipated finding was the value added effect for the active learning approach during the laboratory session for learning about the variables to be measured. To our knowledge, this type of finding has not been reported in the literature about active learning to date. This enhanced effect may be due to the planning required by students before and during the laboratory session featuring the active learning approach; however, it could be due to the focus of that particular laboratory session. The opportunity to collect data from only two laboratory sessions may have influenced this study's findings. Unfortunately, it was not practical to use a case-crossover study design where one-half of students would be asked to perform the same experiment with active learning while the other half used the traditional method.

As anticipated due to the unique features of performing a scientific experiment in a laboratory setting (Bowen, 2017; Cavinato, 2017; Parappilly, 2013; Schiemann, 2006; Thompson, Caroll, \& Laibe, 2013), the qualitative findings indicate that students were making relevant anticipatory and reflective statements about their learning before and after the experiment. In both laboratory sessions, students were aware of the need to plan carefully and be aware of the experiment's features that represented the variables they were studying. However, performing correct procedures was featured prominently, and designing an experiment was uniquely in students' responses for the active learning laboratory session. These finding may also be interpreted to suggest that an active learning approach may stimulate students to think more about their thinking while planning for, and reflecting about a science experiment conducted during an undergraduate pharmaceutical science laboratory session. These findings support the use of active learning strategies may be useful to address the programmatic outcomes established by the accreditation bodies such as those in the USA (Accreditation Council for Pharmacy Education, 2016) and Australia/New Zealand (Australian Pharmacy Council, 2020) regarding the development of students' foundation sciences knowledge that conducting an experiment.

When viewed together, the findings from this study add weight to the argument that an active learning approach may be a more effective method of instruction for developing students understanding of foundation pharmaceutical sciences knowledge in pharmaceutical science laboratory settings than using a traditional method. These findings are also consistent with results reported from other pharmacy education studies featuring active learning
(Kangwantas et al., 2017; Kiersma et al., 2009; Meng et al., 2019; Ross et al., 1999).

The decision to perform this study with third-year undergraduate pharmacy students during their third and fourth laboratory session of their course, may have influenced the data in favour of supporting this study's claim about active learning being more effective than traditional methods. As Kirschner and colleagues (2009) remind us, learning requires students to access long-term memory, therefore, students need to be equipped with prior foundational knowledge before they can apply that knowledge to perform an experiment to answer a research question. The laboratory classes were not designed with additional assessment activities to gather information about the impact the active learning session had on student outcomes, versus the traditional session(s), therefore, the study data are presented in isolation from other assessment data. Additionally, the questions were not tested on a student cohort prior to administration of the questions and so improvement in the language used may be possible. Future work might explore the students' conceptions of key pharmaceutical terminology such as dissolution and disintegration as well as measurement concepts related to reliability and validity. More data could be gathered from subsequent cohorts of students to gain a sufficient sample size to explore possible relationships among demographics, course achievement and quantification of the time spent by students to prepare for each laboratory session. Students could also be involved in reviewing the scoring rubrics developed to interpret their responses. The focus of this study on experimental knowledge draws our attention to this aspect of learning when using an active learning instructional approach at the expense of other relevant aspects such as collaboration and communication. We acknowledge that there may be other times where an active learning approach may not be suitable such as learn how to follow instructions when they are provided, however, those times were not the focus of this study. The authors have interpreted these findings cautiously because, as Sfard (1998) reminds, robust learning methods include a balance of participation and acquisition learning opportunities for students.

\section{Conclusion}

The findings of this study indicate that an active learning approach in a pharmaceutical science laboratory setting appears to be useful to enhance students' experimental knowledge and appears to have a positive impact on student learning. Mixed methods allowed two sources of 
data to be combined and produce a meaningful synthesis of students' experimental knowledge about the two ways they could learn about and perform a pharmaceutical science experiment. Findings from this study suggest that an active learning approach may have a greater magnitude of impact on student learning than traditional experiment methods. This study will be of interest to pharmacy educators interested in developing active learning techniques that address experimental knowledge learning outcomes in their pharmaceutical science laboratories with their students.

\section{Acknowledgements}

The authors thank the 2017 cohort of third-year Bachelor of Pharmacy students at the University of Otago for their willingness to participate in this study; and appreciate the willingness of the laboratory demonstrators, S. Streck, S. Yarragudi and $\mathrm{H}$. Vu for their participation in this study. Anakin and McDowell are grateful to J. Van Dijk for assistance with data entry. The authors also acknowledge $\mathrm{Dr}$ C. Thompson, Monash University, Australia for initial discussions about this study.

\section{References}

Accreditation Council for Pharmacy Education. (2016). Accreditation standards and key elements for the professional program in pharmacy leading to the doctor of pharmacy degree. Chicago, IL: Accreditation Council for Pharmacy Education (online). Available at: https://www.acpe-accredit.org/

Arthur, P., Ludwig, M., Castelli, J., Kirkwood, P., \& Attwood, P. (2016). Prepare, Do, Review: A skills-based approach for laboratory practical classes in biochemistry and molecular biology. Biochemistry and Molecular Biology Education, 44(3), 276-287. https://doi.org/10.1002/bmb.20951

Australian Pharmacy Council. (2020). Accreditation Standards for Pharmacy Programs in Australia and New Zealand 2020 Performance Outcomes Framework. Canberra, Australia: Australian Pharmacy Council. Available at: https://www.pharmacycouncil.org.au/resources/pharmacy-program-standards/

Biggs, J.B., \& Collis, K.F. (1982). Evaluating the quality of learning: The SOLO taxonomy (Structure of the Observed Learning Outcome). New York, NY: Academic Press.

Cavinato, A.G. (2017). Challenges and successes in implementing active learning laboratory experiments for an undergraduate analytical chemistry course. Analytical and Bioanalytical Chemistry, 409(6), 1465-1470. https://doi.org/10.1007/s00216-016-0092-x

Cohen, J. (1988). Statistical power analysis for the behavioural sciences. ( $2^{\text {nd }}$ ed.). New York, NY: Erlbaum.

Creswell, J.W. \& Creswell, J.D. (2018). Research design: Qualitative, quantitative, and mixed methods approaches. (5th ed.). Thousand Oaks, CA: Sage.
Freeman, S., Eddy, S.L., McDonough, M., Smith, M.K., Okoroafor, N., Jordt, H., \& Wenderoth, M.P. (2014). Active learning increases student performance in science, engineering, and mathematics. Proceedings of the National Academy of Sciences, 111(23), 8410-8415. https://doi.org/10.1073/pnas.1319030111

Gleason, B.L., Peeters, M.J., Resman-Targoff, B.H., Karr, S., McBane, S., Kelley, K., Thomas, T., \& Denetclaw, T.H. (2011). An active-learning strategies primer for achieving ability-based educational outcomes. American Journal of Pharmaceutical Education, 75(9), 1-12. https://doi.org/10.5688/ajpe759186

Hallgren, K.A. (2012). Computing inter-rater reliability for observational data: an overview and tutorial. Tutorials in Quantitative Methods for Psychology, 8(1), 23-34.

International Pharmaceutical Federation. (2016). Global vision for education and workforce: Presented at the global conference on pharmacy and pharmaceutical sciences education (online). Available from: https://www.fip.org/files/content/pharmacy-education/fip-education/global-vision-for-education.pdf

International Pharmaceutical Federation. (2017). Nanjing statements on pharmacy and pharmaceutical sciences education (online). Available from: https://www.fip.org/files/content/pharmacyeducation/fip-education/nanjing-statements.pdf

Kangwantas, K., Pongwecharak, J., Rungsardthong, K., Jantarathaneewat, K., Sappruetthikun, P., \& Maluangnon, K. (2017). Implementing a flipped classroom approach to a course module in fundamental nutrition for pharmacy students. Pharmacy Education, 17(1), 329-34.

Kiersma, M.E., Darbishire, P.L., Plake, K.S., Oswald, C., \& Walters, B.M. (2009). Laboratory session to improve first-year pharmacy students' knowledge and confidence concerning the prevention of medication errors. American journal of pharmaceutical education, 73(6), 99. https://doi.org/10.5688/aj730699

Kirschner, P.A., Sweller, J., \& Clark, R.E. (2006). Why minimal guidance during instruction does not work: An analysis of the failure of constructivist, discovery, problem-based, experiential, and inquirybased teaching. Educational Psychologist, 41(2), 75-86. https:// doi.org/10.1207/s15326985ep4102 1.

Meng, X., Yang, L., Sun, H., Du, X., Yang, B., \& Guo, H. (2019). Using a Novel Student-centered Teaching Method to Improve Pharmacy Student Learning. American Journal of Pharmaceutical Education, 83(2), 171-179. https://doi.org/10.5688/aipe6505

Ross, W.H., Lawrence, L.W., Reddy, I.K., Dick, R.M., Roane, D.S., Schulte, M.K., Khan, M.A., Olivier, K.J., \& Sponberg, L.A. (1999). Implementation of a new course with a focus on active learning through integrated curricular approach: pharmacy care laboratory I. Journal of Pharmacy Teaching, 7(2), 15-34.

Schiemann, G. (2006). Experimental knowledge and the theory of producing it: Hermann von Helmholtz. In: U. Feest, G. Hon, H-J. Rheingerger, J. Schickore, \& F. Steinle (Eds.). Experimental knowledge. Berlin, Germany: Max Plank Institute for the History of Science.

Scott, P.H., Veitch, N.J., Gadegaard, H., Mughal, M., Norman, G., \& Welsh, M. (2018). Enhancing theoretical understanding of a practical biology course using active and self-directed learning strategies. Journal of Biological Education, 52(2), 184-195. https:// doi.org/10.1080/00219266.2017.1293557

Sfard. A. (1998). On two metaphors for learning and the dangers of choosing just one. Educational Researcher, 27(2), 4-13. https:// doi.org/10.3102/0013189X027002004 
Stallings, W.M., \& Gillmore, G.M. (1971). A note on "accuracy" and "precision". Journal of Educational Measurement, 8(2), 127-129. https://www.jstor.org/stable/1433969

Thomas, D.R. (2006). A general inductive approach for analyzing qualitative evaluation data. American Journal of Evaluation, 27(2), 237-246. https://doi.org/10.1177/1098214005283748

Thompson, C.D., Carroll, M.R., \& Laibe, F. (2013). The IDEA Experiments: Enabling genuine inquiry and design skills in the undergraduate chemistry laboratory. In: P. Newitt (Ed.). Proceedings of the Australian Conference on Science and Mathematics Education, Australian National University, p. 65, Available from: https://openjournals.library.sydney.edu.au/index.php/IISME/issue/view/606.

Thompson, C., Rayner, G., Barratt, C., Hughes, T., \& Kirkup, L. (2014). Taking inquiry-oriented learning to the teaching coalface: $A$ good practice booklet for practitioners. Sydney, Australia: Office for Teaching and Learning, Department of Industry, Innovation, Science, Research and Tertiary Education.

\section{Appendices}

\section{Appendix A}

(Active Learning) Experiment instructions for Laboratory Manual Lab 3: Effect of excipients on in vitro dissolution rate of tablets Part A

Determine the effects of hydroxypropyl cellulose and stearic acid on the dissolution rate of theophylline

\section{Methods}

In your lab group, design an experiment to determine the dissolution rate of compressed disks of theophylline (anhydrous) alone and disks co-compressed with 5, 10, 20 and 40\% hydroxypropyl cellulose (HPC) or 5, 10, 20 and $40 \%$ stearic acid.

Use the space below to plan your experiment with the equipment available in the teaching lab. Consider samples to be tested, sampling time, sample volume, set up of dissolution equipment etc.

Note: From your previous study and the pre-lab reading, it is expected that you are familiar with how to perform a dissolution experiment.

Once your plan has been approved by a demonstrator, you will conduct your experiment in your lab group using one of the excipients.

(Traditional) Experiment instructions for Laboratory Manual Lab 3: Effect of excipients on in vitro dissolution rate of tablets

Part A

Determine the effects of hydroxypropyl cellulose and stearic acid on the dissolution rate of theophylline

\section{Methods}

Determine the dissolution rate of compressed disks of theophylline (anhydrous) alone and disks co-compressed with 5, 10, 20 and $40 \%$ hydroxypropyl cellulose (HPC) and stearic acid.
Each group of six students will measure the dissolution rates of two theophylline alone disks (2 disks) and one each of 5, 10, 20 and $40 \%$ HPC or stearic acid (4 disks).

\section{Dissolution}

1. Insert the compressed discs in the rotating tablet holder and adjust the height of the tablet holders so they are the correct distance $(2.5 \mathrm{~cm})$ from the bottom of the beaker. The height can be checked with the special tool.

2. Check that the stirring speed is set at $\mathbf{2 0 0} \mathbf{~ r p m}$.

3. Transfer $400 \mathrm{~mL}$ of the $\mathrm{pH} 5.5$ buffer (deaerated and preequilibrated to $37 \mathrm{C}$ ) to each of the dissolution beakers and place in the dissolution apparatus.

4. Check the temperature of the medium in each of the dissolution containers. When the temperature reaches $37.0+/-2.0 \mathrm{C}$ you may start the experiment.

5. Lower the disks into the media. Check no air bubbles have settled on the under-surface of the disk. If so, dislodge them with the right-angle wire provided.

6. Start the stirrers and the clock.

7. After $\mathbf{2}$ minutes, take your first samples. Try to synchronise sampling from all containers if possible. Quickly, using pipettors, remove $1.0 \mathrm{~mL}$ from each container from a position near the edge of the container and about $1 \mathrm{~cm}$ from the surface of the liquid. (You do not need to change the pipettor tip providing you use the same tip for all samples from one beaker only).

8. Continue to take $\mathbf{1 . 0} \mathrm{mL}$ samples at $\mathbf{4}$ min intervals. Take a total of 6 samples.

9. When finished, inspect the tablet disks. Describe what you observe?

\section{Analysis for theophylline content}

Theophylline will be analysed by UV spectrometry at $268 \mathrm{~nm}$.

Make the appropriate dilution of your samples with buffer to ensure they are within range of the standard curve. A 1 in 10 dilution is suggested.

Record absorbance of your diluted samples at $\mathbf{2 6 8} \mathbf{~ n m}$; tabulate your results.

\section{Calculations \& graphical treatment of results}

Calculate the concentration of theophylline present in each sample using the standard curve provided. Convert this to $\mathbf{m g}$ theophylline dissolved and enter into the Table. [Note: the excipient does not interfere with the UV spectrum of theophylline.]

Prepare graphs showing $\mathbf{m g}$ theophylline dissolved versus time for each excipient.

Inspect the profiles for linearity.

Calculate the dissolution rates $(\mathrm{mg} / \mathrm{min})$ from the gradient of each profile if linear. Convert these rates to $\mathrm{mg} / \mathrm{min} / \mathrm{cm}^{2}$. 